\title{
Mutual Correlation in the Shock Wave Geometry
}

\author{
Xiao-Xiong Zeng $\mathbb{D},{ }^{1}$ Hong-Bo Shao, ${ }^{2}$ Ling $\mathrm{Li}^{3}{ }^{3}$ and Xian-Ming Liu $\mathbb{D}^{4}$ \\ ${ }^{1}$ School of Material Science and Engineering, Chongqing Jiaotong University, Chongqing 400074, China \\ ${ }^{2}$ College of Science, Agricultural University of Hebei, Baoding 071000, China \\ ${ }^{3}$ School of Mathematics, Sichuan University of Arts and Science, Dazhou 635000, China \\ ${ }^{4}$ School of Science, Hubei University for Nationalities, Enshi, 445000, China
}

Correspondence should be addressed to Xiao-Xiong Zeng; xxzengphysics@163.com

Received 11 March 2018; Revised 13 June 2018; Accepted 25 June 2018; Published 13 August 2018

Academic Editor: Elias C. Vagenas

Copyright ( 2018 Xiao-Xiong Zeng et al. This is an open access article distributed under the Creative Commons Attribution License, which permits unrestricted use, distribution, and reproduction in any medium, provided the original work is properly cited. The publication of this article was funded by SCOAP S $^{3}$

\begin{abstract}
We probe the shock wave geometry with the mutual correlation in a spherically symmetric Reissner-Nordström AdS black hole on the basis of the gauge/gravity duality. In the static background, we find that the regions living on the boundary of the AdS black holes are correlated provided the considered regions on the boundary are large enough. We also investigate the effect of the charge on the mutual correlation and find that the bigger the value of the charge is, the smaller the value of the mutual correlation will be. As a small perturbation is added at the AdS boundary, the horizon shifts and a dynamical shock wave geometry form after long time enough. In this dynamic background, we find that the greater the shift of the horizon is, the smaller the mutual correlation will be. Especially for the case that the shift is large enough, the mutual correlation vanishes, which implies that the considered regions on the boundary are uncorrelated. The effect of the charge on the mutual correlation in this dynamic background is found to be the same as that in the static background.
\end{abstract}

\section{Introduction}

Butterfly effect is ubiquitous phenomenon in physical systems. One progress on this topic recent years is that it also can be addressed in the context of gravity theory [1-15] with the help of the AdS/CFT correspondence [16-18]. In this framework, one can define the so-called thermofield double state on the boundary of an eternal AdS black hole [19]. As a small perturbation with energy $E$ is added along the constant $\mu$ trajectory in the Kruskal coordinate to one of the boundary at early time $t_{w}$, one finds that a bound of infinite energy accumulates near the horizon and a shock wave geometry forms at $t=0$, which is the so-called butterfly effect in the AdS black holes [20]. The evolution of the shock wave is dual to the evolution of the thermofield double state according to the intercalation of the AdS/CFT correspondence. The mutual information, defined by

$$
M(A, B) \equiv S(A)+S(B)-S(A \cup B),
$$

is often used to probe the effect of the shock wave on the entanglement of the subsystems $A$ and $B$ living on the boundary [20], where $S(A), S(B)$ are the entanglement entropy of the space-like regions on $A$ and $B$, which can be calculated by the area of the minimal surface proposed by Ryu and Takayanagi [21], while $S(A \cup B)$ is the entanglement entropy of a region which cross the horizon and connects $A$ and $B$.

There are two important quantities characterizing the butterfly effect. One is the scrambling time, which takes the universal form $[20]$ :

$$
t_{\star}=\beta \log S,
$$

where $S$ is the black hole entropy and $\beta$ is the inverse temperature. The scrambling time is the time when the mutual information between the two sides on $A$ and $B$ vanishes. The other is the Lyapunov exponent $\lambda_{L}$, which has the following bound [22]:

$$
\lambda_{L} \leq \frac{2 \pi}{\beta}
$$

and the saturation of this bound has been suggested as the criterion on whether a many-body system has a holographic 
dual with a bulk theory [22]. A remarkable example that saturates this bound is the Sachdev-Ye-Kitaev model [22].

In the initial investigation, the dual black hole geometry is the nonrotating BTZ black hole [20]. The area of the minimal surface equals the length of the geodesic on the boundary. The mutual information thus is defined by the geodesic length. In this paper, we intend to study butterfly effect in the 4dimensional Reissner-Nordström AdS black holes. Though the area of the minimal surface does not equal the length of the geodesic, we want to explore whether there is a quantity defined by the length of the geodesic that can still probe the butterfly effect. We define this quantity as mutual correlation:

$$
I(A, B) \equiv L(A)+L(B)-L(A \cup B)
$$

in which $A$ and $B$ are two points on the left and right boundaries, $L(A), L(B)$ are the space-like geodesic that go through points $A$ and $B$, respectively, and $L(A \cup B)$ is the geodesic length cross the horizon and connects $A$ and $B$. The results are not expectable since we cannot view simply the mutual correlation as the spatial section of the mutual information by fixing some of the transverse coordinates. The metric components of the transverse coordinates are not one but the functions of the radial coordinate $r$ so that they have contributions to the area of the minimal surface.

In the 4-dimensional space-time, though the geodesic length does not equal the area of the minimal surface, it has been shown that both the geodesic length and area of the minimal surface, which are dual to the two point correlation function and entanglement entropy respectively, are nonlocal probes and have the same effect as they are used to probe the thermalization behavior and phase transition process [23-40]. Thus it is interesting to explore whether the mutual correlation can probe the butterfly effect as the mutual information for both of them are defined by the nonlocal probes.

In [1], the author has probed the shock wave geometry with mutual information in the 4-dimensional plane symmetric Reissner-Nordström AdS black branes. They have obtained some analytical results approximately and found that for large regions the mutual information is positive in the static black hole, and the mutual information will be disrupted as a small perturbation is added in dynamic background. In this paper, we will employ the mutual correlation to probe the shock wave geometry in the 4-dimensional spherically symmetric Reissner-Nordström AdS black holes. Our motivation is twofold. On one hand, we intend to give the exact numeric result between the size of the boundary region and mutual correlation as well as the perturbation and mutual correlation. One the other hand, we intend to explore how the charge affects the mutual correlation in cases without and with a perturbation. Both cases have not been reported previously in [1].

Our paper is outlined as follows. In Section 1, we will construct the shock wave geometry in the Reissner-Nordström AdS black holes. In Section 2, we will study the mutual correlation in the static background. We concentrate on the effect of the boundary separation and charge on the mutual correlation. In Section 3, we will probe the butterfly effect with the mutual correlation in the dynamical background. We concentrate on studying the effect of the perturbation and charge on the mutual correlation. The conclusion and discussion are presented in Section 4. Hereafter in this paper we use natural units $(G=c=\hbar=1)$ for simplicity.

\section{Shock Wave Geometry in the Reissner-Nordström AdS Black Holes}

Starting from the action,

$$
S=-\frac{1}{16 \pi G} \int d^{d+1} x \sqrt{g}\left(\mathscr{R}+\frac{d(d-1)}{\ell^{2}}-\frac{1}{4} F_{\mu \nu} F^{\mu \nu}\right),
$$

one can get the Reissner-Nordström AdS black holes solution. For the case $d=3$, we have

$$
d s^{2}=-f(r) d t^{2}+\frac{d r^{2}}{f(r)}+r^{2}\left(d \theta^{2}+\sin ^{2} \theta \phi^{2}\right),
$$

in which $f(r)=1-2 M / r+Q^{2} / r^{2}+r^{2}$, where $M$ is the mass and $Q$ is the charge of the black hole.

In order to discuss the butterfly effect of a black hole, one should construct the shock wave geometry in the Kruskal coordinate firstly. We will review the key procedures and give the main results as done in [20] for the consistency of this paper though there have been some discussions on this topic.

The event horizon, $r_{h}$, of the black hole is determined by $f\left(r_{h}\right)=0$. With the definition of the surface gravity, $\kappa=\left.f(r)^{\prime}\right|_{r_{h}} / 2$, we also can get the Hawking temperature $T=\kappa / 2 \pi$, which is regarded as the temperature of the dual conformal field theory according to the AdS/CFT correspondence. In the Kruskal coordinate system, the metric in (6) can be rewritten as

$$
d s^{2}=\frac{1}{\kappa^{2}} \frac{f(r)}{\mu \nu} d \mu d \nu+r^{2}\left(d \theta^{2}+\sin ^{2} \theta \phi^{2}\right),
$$

in which

$$
\begin{gathered}
\mu= \pm e^{-\kappa U}, \\
\nu=\mp e^{\kappa V}, \\
\mu \nu=-e^{2 \kappa r_{\star}}, \\
\frac{\mu}{\nu}=-e^{-2 \kappa t},
\end{gathered}
$$

where $U=t-r_{\star}, V=t+r_{\star}$, are the Eddington coordinate, which are defined by the tortoise coordinate $r_{\star}=\int(d r / f(r))$. We will suppose $\mu<0, v\rangle 0$ at the right exterior as in [20]. As $r$ approaches to the event horizon and boundary, we know $r_{\star}$ approaches to $-\infty$ and 0 , respectively. Thus from (9), we know that the event horizon and boundary locate at $\mu \nu=0$ and $\mu \nu=-1$, respectively.

Next we will check how the space-time changes as a small perturbation with asymptotic energy $E$ is added on the left boundary at time $t_{w}$ following a constant $\mu$ trajectory. We 
label the Kruskal coordinate on the left side and right side as $\mu_{L}, \nu_{L}$ and $\mu_{R}, v_{R}$. The constant $\mu$ trajectory propagation of the perturbations implies

$$
\mu_{L}=\mu_{R}=e^{-\kappa t_{w}}
$$

To find the relation between $v_{L}$ and $v_{R}$, we will employ the following relation:

$$
\begin{aligned}
& \mu_{L} \nu_{L}=-e^{2 \kappa_{L} r_{\star L}}, \\
& \mu_{R} \nu_{R}=-e^{2 \kappa_{R} r_{\star R}} .
\end{aligned}
$$

Generally speaking, $\kappa_{L}=\kappa_{R}=\kappa$ for the energy $E$ of the perturbation is much smaller than that of the black hole mass $M$. On the other hand, we are interested in the case $t_{w} \longrightarrow \infty$, which implies $r \longrightarrow r_{h}$. In this case, we can approximate $r_{\star} \approx(1 / 2 \kappa)\left(\log ^{r-r_{h}}+c\right)$ for there is a relation $f(r)=f^{\prime}\left(r_{h}\right)\left(r-r_{h}\right)+\cdots$. In this case, $e^{2 \kappa r_{\star}}=C\left(r-r_{h}\right)$, here $C=e^{c}$. So we have the identification

$$
v_{L}=v_{R}+C e^{\kappa t_{w}}\left(r_{h L}-r_{h R}\right) \equiv v_{R}+h,
$$

where we have used the relation $C_{L}=C_{R}=C$. From (12), we know that there is a shift in the Kruskal coordinate $v$ as the small perturbation is across the $\mu=0$ horizon of the black hole. For computations, the shift in $v$ is often written as $\nu \longrightarrow$ $\nu+h(\theta) \Theta(\mu)$, where $\Theta(\mu)$ is a step function. In this case, (7) changes into a standard shock wave:

$$
\begin{aligned}
d s^{2}= & A(\mu \nu) d \mu d \nu-A(\mu \nu) h(\theta) \delta(\mu) d \mu^{2} \\
& +B(\mu \nu)\left(d \theta^{2}+\sin ^{2} \theta \phi^{2}\right)
\end{aligned}
$$

in which we have used the relation $\Theta(\mu)^{\prime}=\delta(\mu)$ and the replacement

$$
\begin{aligned}
& A(\mu \nu)=\frac{1}{\kappa^{2}} \frac{f(r((\mu \nu))}{\mu \nu}, \\
& B(\mu \nu)=r(\mu \nu)^{2} .
\end{aligned}
$$

The Kruskal diagram for the perturbed space-time is shown in Figure 1.

\section{Mutual Correlation in the Static Reissner-Nordström AdS Black Holes}

In this section, we will investigate the mutual correlation in the static background. Our objective is to explore whether the boundary regions of the AdS black holes are correlated so that we can investigate the effect of the shock wave on the mutual correlation in the next section.

As depicted in Figure 1, an eternal black hole has two asymptotically AdS regions, which can be holographically described by two identical, noninteracting copies of the conformal field theory. One thus can define the so-called thermal double state and study their entanglement and correlation. Our objective is to compute the mutual correlation of point $A$ on the left asymptotic boundary and its partner $B$ on the right asymptotic boundary. We will let $A=B$ so that the left and right boundaries are identical. For the spherically symmetric black holes in this paper, the AdS boundary is a 2-dimensional sphere with finite volume. In light of the symmetry of $\phi$ direction, we will use $\theta$ to parameterize the geodesic length between any two points on the boundary, named $\theta_{1}, \theta_{2}$.

On the left boundary, the geodesic length that goes through point $A$ with boundary separation $\theta_{0}$ is

$$
L_{A}=\int d S=\int d \theta \sqrt{f^{-1} r^{\prime 2}+r^{2}}
$$

where $r^{\prime}=d r / d \theta$. When regarding the integrand in (15) as the Lagrangian, we can define a conserved quantity associated with translations in $\theta$, that is,

$$
\frac{r^{2}}{\sqrt{r^{2}+f^{-1} r^{\prime 2}}}=r_{\min }
$$

where $r_{\min }$ is the turning point of the surface where $d r / d \theta=$ $\left(\theta^{\prime}\right)^{-1}=0$. According to the symmetry, it locates at $\theta=\theta_{0} / 2$. With (16), $\theta_{0}$ can be written as

$$
\theta_{0}=\int d \theta=2 \int_{r_{\min }}^{\infty} \frac{d r}{r \sqrt{f}} \frac{1}{\sqrt{\left(r / r_{\min }\right)^{2}-1}} .
$$

The geodesic length also can be rewritten as

$$
L_{A}=2 \int_{r_{\min }}^{\infty} d r \frac{1}{\sqrt{f}} \frac{1}{\sqrt{1-\left(r_{\min } / r\right)^{2}}} .
$$

Since $B$ is identified with $A, L_{B}$ thus takes the same form as $L_{A}$ provided the two points on the boundary located at the same place. As stressed in the introduction, we will employ the mutual correlation to study the correlation between points $A$ and $B$. Thus our next step is to find $L_{A \cup B}$, which is the geodesic length connecting the left point and right point by passing through the horizon of the black hole, where $\theta^{\prime}=0$. The total length, including both sides of the horizon, can be expressed as

$$
L_{A \cup B}=4 \int_{r_{h}}^{\infty} d r \sqrt{f^{-1}} .
$$

Putting all these results together, the mutual correlation can be expressed as

$$
I\left(\theta_{0}\right)=4 \int_{r_{\min }}^{\infty} d r \frac{1}{\sqrt{f}} \frac{1}{\sqrt{1-\left(r_{\min } / r\right)^{2}}}-4 \int_{r_{h}}^{\infty} d r \frac{1}{\sqrt{f}}
$$

From Figure 2, one can read off the relation between the mutual correlation and the position of the turning point $r_{\min }$. From this figure, we know that $I\left(\theta_{0}\right)$ decreases as the value of $r_{\text {min }}$ becomes smaller, and $I\left(\theta_{0}\right)$ vanishes as $r_{\text {min }}$ is larger than $r_{h}$ a little. Especially, as $r_{\min } \longrightarrow r_{h}$ the mutual correlation will diverge. That is to say, $r_{\min }$ can not penetrate into the black hole, which was also observed in [41] where the properties of the geodesic length have been investigated extensively. 


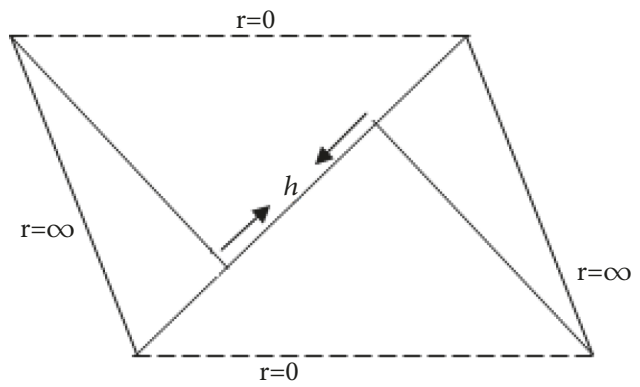

Figure 1: Penrose diagrams for an eternal black hole with a perturbation.

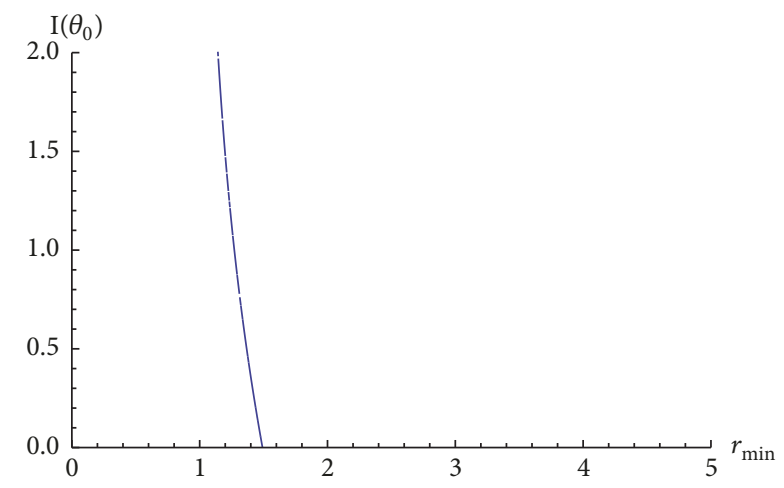

FIgURE 2: Relation between $I\left(\theta_{0}\right)$ and $r_{\min }$ for the case $Q=0.5$.

We also can study the effect of $Q$ on the mutual correlation $I\left(\theta_{0}\right)$, which is shown in Figure 3. From this figure, we know that $I\left(\theta_{0}\right)$ decreases as $Q$ grows for a fixed $r_{\text {min }}$. There is also a critical charge $Q_{c}$ where the mutual correlation vanishes, which means that there is no correlation between the paired subregions we considered. For different $r_{\text {min }}$, the value of the critical charge is different. As $r_{\min }$ increases, the value of the critical charge decreases. For a fixed $Q$, we find that the mutual correlation is smaller for greater $r_{\min }$.

We are interested in how the boundary separation $\theta_{0}$ affects the mutual correlation, especially to each extent, the mutual correlation vanishes. We thus should express the mutual correlation as a function of the boundary separation. Substituting (17) into (20), we obtain

$$
\begin{aligned}
I\left(\theta_{0}\right)= & 2 \theta_{0} r_{\min }+4 \int_{r_{\min }}^{\infty} d r \frac{1}{\sqrt{f}} \sqrt{1-\left(\frac{r_{\min }}{r}\right)^{2}} \\
& -4 \int_{r_{h}}^{\infty} d r \frac{1}{\sqrt{f}} .
\end{aligned}
$$

From Figure 2, we know that $I\left(\theta_{0}\right)$ will vanish as $r_{\min } \simeq r_{h}$. With this approximation, the critical value of the boundary separation in (21) can be expressed as

$$
\theta_{0 c}=\frac{2}{r_{h}}\left[\int_{r_{h}}^{\infty} d r \frac{1}{\sqrt{f}}\left(1-\sqrt{1-\left(\frac{r_{h}}{r}\right)^{2}}\right)\right] .
$$

With (22), we can discuss how the critical value of the boundary separation $\theta_{0 c}$ changes with respect to the horizon

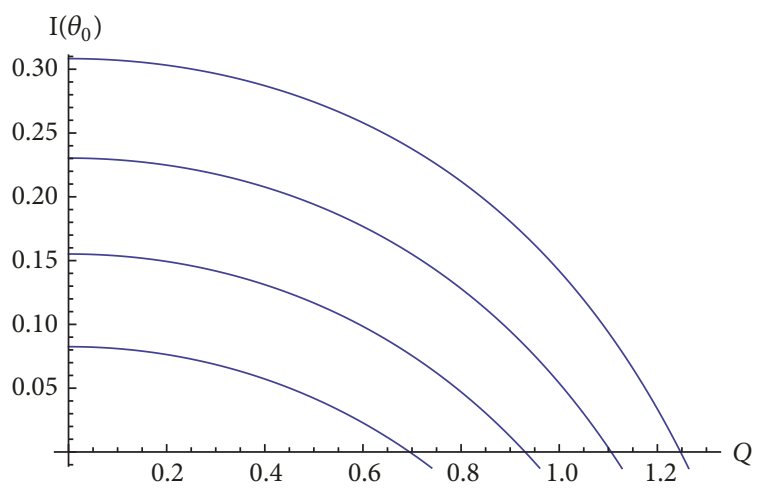

Figure 3: Relation between $I\left(\theta_{0}\right)$ and $Q$. Curves from top to down represent $r_{\text {min }}$ increases from 1.42 to 1.48 with step 0.02 . For both cases, we have set $r_{h}=1$.

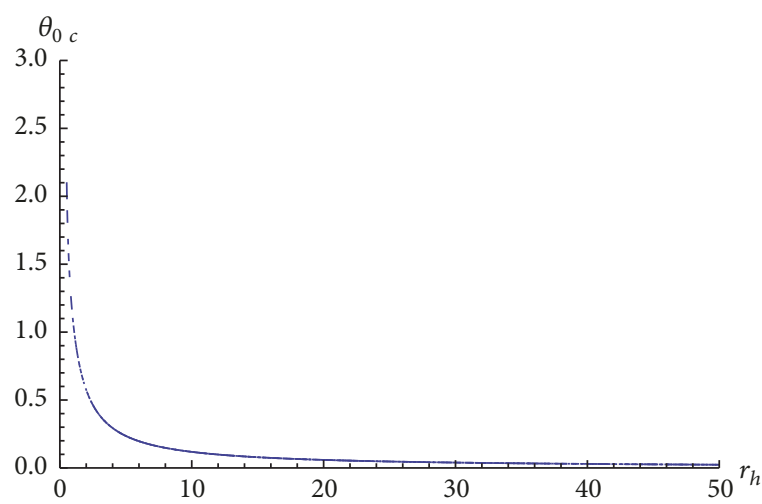

Figure 4: Relation between $\theta_{0 c}$ and $r_{h}$ for the case $Q=0.5$.

$r_{h}$. From Figure 4 , we know that $\theta_{0 c}$ decreases as $r_{h}$ increases. For large enough $r_{h}, \theta_{0 c}$ vanishes. In the small $r_{h}$ region, $\theta_{0 c}$ changes sharply as $r_{h}$ increases. Figure 5 is helpful for us to understand Figure 4. As we addressed previously, $\theta_{0 c}$ is obtained at $r_{h} \approx r_{\text {min }}$. The relation between $\theta_{0 c}$ and $r_{h}$ thus is similar to that of $\theta_{0}$ and $r_{\text {min }}$. As $r_{\text {min }}=\infty$, the geodesic length and further the boundary separation approach to zero naturally.

We already know that bigger $r_{\min }$ actually corresponds to smaller separation on the boundary. Therefore, Figure 3 also indicates that smaller subregions have smaller mutual correlation between them, which is consistent with the physical intuition.

\section{Probe the Shock Wave Geometry via Mutual Correlation}

As a small perturbation is added from the left boundary, there is a shift in the $v$ direction for enough long time $t_{w}$. A shock wave geometry forms and the passage connecting the left region and right region, namely, the wormhole, is disrupted. In this section, we intend to investigate the effect of the disrupted geometry on the mutual correlation. As in Section 3, we suppose point $A$ belongs to the left asymptotic boundary and its identical partner $B$ belongs to the right asymptotic boundary. At $t=0$, geodesic lengths $L_{A}$ and $L_{B}$ 


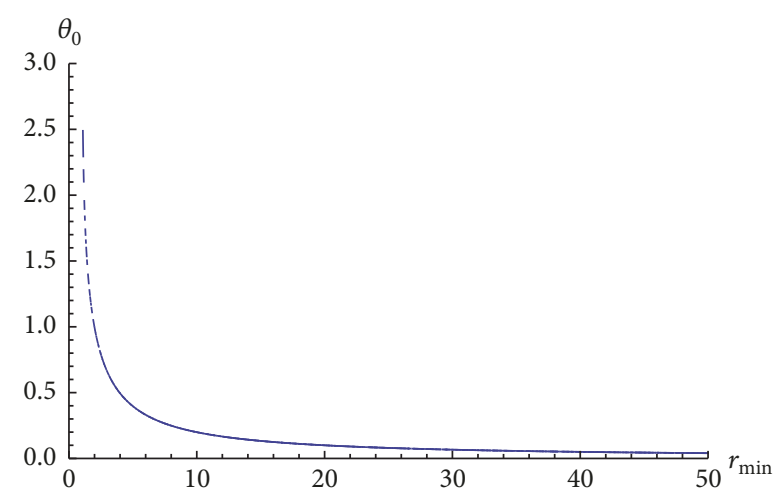

FIgURE 5: Relation between $\theta_{0}$ and $r_{\text {min }}$ for the case $Q=0.5$.

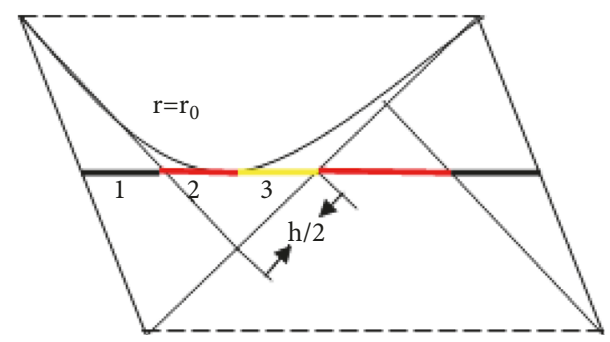

Figure 6: The Penrose diagram and geodesic length (horizontal colourful line) in the shock wave geometry. The left half of the surface is divided into three segments, labeled by black line, red line, and yellow line. The smallest value of $r$ attained by the surface is $r=r_{0}$, which marks the division between 2 and 3 .

are unaffected by the shock wave because they do not cross the horizon. However, the quantity $L_{A \cup B}$ will be affected by the shock wave for it stretches across the wormhole, which is shown in Figure 6.

In light of the identification between $A$ and $B$ as well as the symmetry of the transverse space, we only should calculate the geodesic length for regions 1, 2, and 3 in Figure 6 for the length of the other part is the same as this part. At a constant $\theta$ surface, the induced metric can be written as

$$
d x^{2}=\left[-f(r)+\frac{1}{f(r)} \dot{r}^{2}\right] d t^{2}+r^{2} \sin ^{2} \theta \phi^{2},
$$

in which we have used $r$ to parameterize the surface and $\dot{r}=$ $d r / d t$. The geodesic length for regions 1, 2, and 3 in Figure 6 is then given by

$$
\bar{L}_{A \cup B}(h)=\int d t \sqrt{-f+f^{-1} \dot{r}^{2}} .
$$

It should be stressed that, in Figure 6, the boundary is a 2-dimensional spherical surface in the Penrose diagram strictly. In this paper, we only consider the geodesic length and neglect the contribution of the $\phi$ direction.

When regarding the integrand in (24) as the Lagrangian, we can define the "Hamiltonian" $\mathscr{H}$ as

$$
\mathscr{H}=\frac{-f}{\sqrt{-f+f^{-1} \dot{r}^{2}}}=\sqrt{-f_{0}},
$$

in which $f_{0}=f\left(r_{0}\right)$ and $r_{0}$ is the radial position behind the horizon that satisfies $\dot{r}=0$. From (25), we know that as $r_{0} \longrightarrow$ $r_{h}, \mathscr{H} \longrightarrow 0$, which correspond to the case that the shock wave is absent for $h \longrightarrow 0$ in this case. With the conservation equation, $t$ coordinate can be written as a function of $r$ :

$$
t(r)= \pm \int \frac{d r}{f \sqrt{1+\mathscr{H}^{-2} f}}
$$

where \pm denote $\dot{r}>0$ and $\dot{r}<0$, respectively. Substituting (26) into (24), we can get a time-independent integrand:

$$
\bar{L}_{A \cup B}(h)=\int d r \frac{1}{\sqrt{\mathscr{H}^{2}+f}} .
$$

With this relation, we will compute the geodesic length that starts at $t=0$ on the left asymptotic boundary and ends at $\nu=h / 2$ on the horizon, namely, the geodesics length of region $1+2+3$ in Figure 6, which can be expressed as

$$
\bar{L}_{A \cup B}(h)=\int_{r_{h}}^{\infty} d r \frac{1}{\sqrt{\mathscr{H}^{2}+f}}+2 \int_{r_{0}}^{r_{h}} d r \frac{1}{\sqrt{\mathscr{H}^{2}+f}} .
$$

The second term contains a prefactor 2 which stems from the fact that the second and third segments in Figure 6 have the same length. The total geodesic length, defined as $L_{A \cup B}(h)$, connecting the left boundary and right boundary thus is

$$
L_{A \cup B}(h)=2 \int_{r_{h}}^{\infty} d r \frac{1}{\sqrt{\mathscr{H}^{2}+f}}+4 \int_{r_{0}}^{r_{h}} d r \frac{1}{\sqrt{\mathscr{H}^{2}+f}} .
$$

It should be stressed that the first segment contains a divergent $h$-independent contribution which must be subtracted as we study it numerically. Considering the contribution of $L_{A}$ and $L_{B}$, the mutual correlation in the shock wave geometry can be expressed as

$$
\begin{aligned}
I\left(h, \theta_{0}\right)= & 4 \int_{r_{\min }}^{\infty} d r \frac{1}{\sqrt{f}} \frac{1}{\sqrt{1-\left(r_{\min } / r\right)^{2}}} \\
& -2 \int_{r_{h}}^{\infty} d r \frac{1}{\sqrt{\mathscr{H}^{2}+f}} \\
& -4 \int_{r_{0}}^{r_{h}} d r \frac{1}{\sqrt{\mathscr{H}^{2}+f}} .
\end{aligned}
$$

Of course, the first term on the right is divergent on the boundary, the contribution from the pure AdS should be subtracted as we calculate it numerically.

For a fixed $r_{h}$, we know that $I\left(h, \theta_{0}\right)$ depends on the location of $r_{0}$. The main objective of this section is to probe the shock wave geometry with the mutual correlation; we thus should find the relation between $I\left(h, \theta_{0}\right)$ and $h$. To proceed, we should find the relation between $h$ and $r_{0}$. 
Firstly, we should find the coordinates of the three segments in Figure 6. The first segment goes from the boundary at $(\mu, v)=(1,-1)$ to $(\mu, v)=\left(\mu_{1}, 0\right)$, in which

$$
\mu_{1}=\exp \left[-\kappa \int_{r_{h}}^{\infty} \frac{d r}{f}\left(1-\frac{1}{\sqrt{1+\mathscr{H}^{-2} f}}\right)\right],
$$

where we have used (9). The second segment stretches from $\left(\mu_{1}, 0\right)$ to $\left(\mu_{2}, v_{2}\right)$ at which $r=r_{0}$. The coordinate $\mu_{2}$ can be determined by the following relation:

$$
\frac{\mu_{2}}{\mu_{1}}=\exp \left[-\kappa \int_{r_{0}}^{r_{h}} \frac{d r}{f}\left(1-\frac{1}{\sqrt{1+\mathscr{H}^{-2} f}}\right)\right] .
$$

The coordinate $v_{2}$ can be determined by choosing a reference surface $r=\bar{r}$ for which $r_{\star}=0$ in the black hole interior. In this case,

$$
\nu_{2}=\frac{1}{\mu_{2}} \exp \left(2 \kappa \int_{\bar{r}}^{r_{0}} \frac{d r}{f}\right)
$$

The third segment stretches from $\left(\mu_{2}, v_{2}\right)$ to $\left(\mu_{3}=0, v_{3}=\right.$ $h / 2$ ). With the relation

$$
\begin{aligned}
\frac{v_{3}}{v_{2}} & =\frac{h}{2 v_{2}}=\exp \left[\kappa \int_{r_{0}}^{r_{h}} \frac{d r}{f}\left(1-\frac{1}{\sqrt{1+\mathscr{H}^{-2} f}}\right)\right] \\
& =\frac{\mu_{1}}{\mu_{2}}
\end{aligned}
$$

we can express $h$ as

$$
h=2 \exp \left(\Pi_{1}+\Pi_{2}+\Pi_{3}\right),
$$

where

$$
\begin{aligned}
& \Pi_{1}=2 \kappa \int_{\bar{r}}^{r_{0}} \frac{d r}{f}, \\
& \Pi_{2}=2 \kappa \int_{r_{0}}^{r_{h}} \frac{d r}{f}\left(1-\frac{1}{\sqrt{1+\mathscr{H}^{-2} f}}\right), \\
& \Pi_{3}=\kappa \int_{r_{h}}^{\infty} \frac{d r}{f}\left(1-\frac{1}{\sqrt{1+\mathscr{H}^{-2} f}}\right) .
\end{aligned}
$$

It is obvious that $h$ depends on the location of $r_{0}$ for fixed $r_{h}$. The relation between $I\left(h, \theta_{0}\right)$ and $h$ is shown in Figure 7. From this figure, we can see that for a fixed charge the relation between $r_{0}$ and $h$ is nonmonotonic. Here we are interested in two locations on the horizontal axis. One is the initial location of the curve where $h$ approaches to infinity, which implies that $h$ is divergent. We label the corresponding horizontal axis of the divergent point as $r_{0 d h}$. The other is the final location of the curve, where $h$ vanishes. Obviously, in

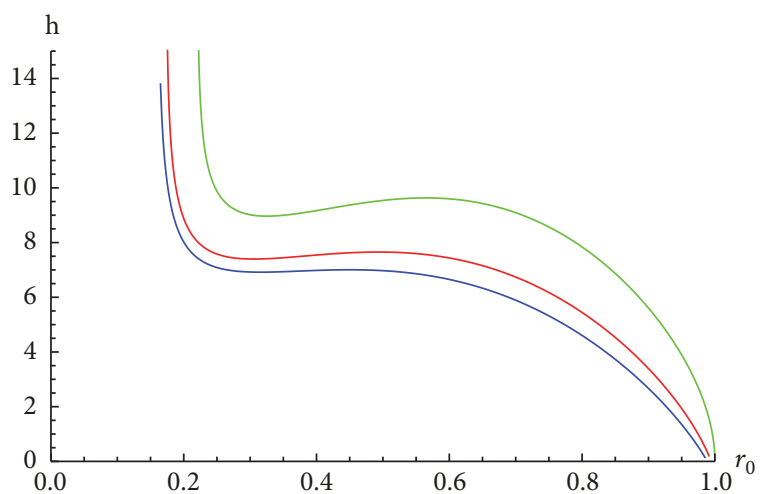

Figure 7: Relation between $h$ and $r_{0}$ for the case $\bar{r}=0.2, r_{h}=1$. The green line, red line, and blue line correspond to $Q=0.5,0.52,0.54$, respectively.

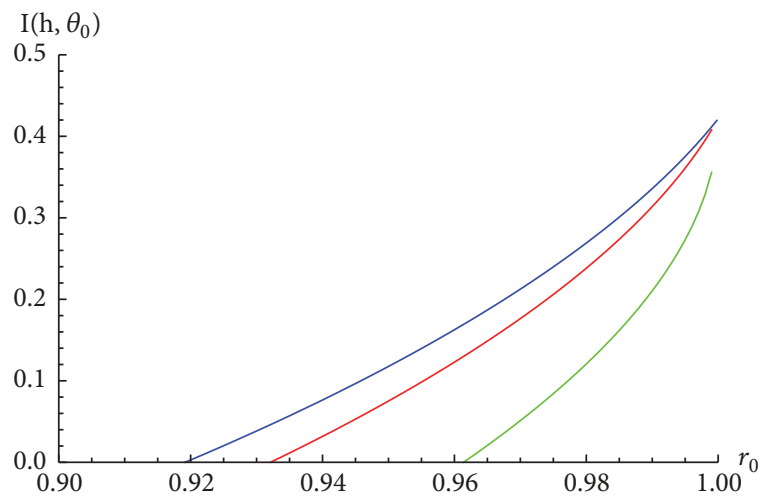

Figure 8: Relation between $I\left(h, \theta_{0}\right)$ and $r_{0}$ for the case $r_{\min }=$ $50, r_{h}=1$. The green line, red line, and blue line correspond to $Q=0.5,0.52,0.54$, respectively.

this case, $r_{0} \longrightarrow r_{h}$. The corresponding horizontal axis of the critical point is labeled as $r_{0 c h}$. In fact, for the plane symmetric black holes, [1] has obtained these results analytically. It was found that, at $r_{0 d h}, \Xi_{3}$ diverges; thus $h$ approaches to infinity. At $r_{0 c h}, h$ vanishes for both $\Xi_{1}$ and $\Xi_{2}$ behaving as $\log \left(r_{h}-r_{0}\right)$. Our results show that these conclusions are still valid for the spherically symmetric black holes. We also investigate the effect of the charge on the shift $h$. We can see that as the charge increases, both the values of the divergent point and critical point become smaller. In addition, we find, for a fixed $r_{0}$, greater value of the charge corresponds to smaller shift $h$, which implies the charge delays the formation of the shock wave geometry.

With (30), we can get the relation between $I\left(h, \theta_{0}\right)$ and $r_{0}$, which is shown in Figure 8. We can see that, for a fixed charge, $I\left(h, \theta_{0}\right)$ increases as $r_{0}$ increases. Especially, there is a critical value of $r_{0}$, where $I\left(h, \theta_{0}\right)$ vanishes. We label the corresponding horizontal axis of the critical point as $r_{0 c i}$. We also investigate the effect of the charge on the critical point $r_{0 c i}$ and find that larger the value of the charge is, the smaller the value of $r_{0 c i}$ will be. For a fixed value of $r_{0}$, the mutual correlation is bigger as the charge $Q$ becomes greater. This seems to contradict with the statements in Section 3 where the 


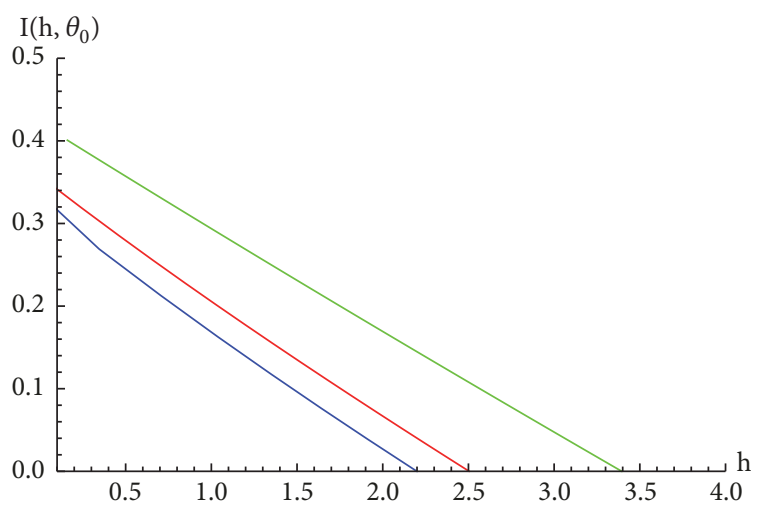

FIGURE 9: Relation between $I\left(h, \theta_{0}\right)$ and $h$ for the case $\bar{r}=0.2, r_{\text {min }}=$ 50. The green line, red line, and blue line correspond to $Q=$ $0.5,0.52,0.54$, respectively.

mutual correlation decreases with respect to the charge. The readers should note that in Section 3 there is no shake wave added in the background. This observation indicates that the dynamical shock wave geometry has dominant impact on the mutual correlation in the shock wave geometry.

Having obtained the relation between $h$ and $r_{0}$ as well as $I\left(h, \theta_{0}\right)$ and $r_{0}$, we can obtain the relation between $I\left(h, \theta_{0}\right)$ and $h$, which is shown in Figure 9. It is obvious that as $h$ increases, $I\left(h, \theta_{0}\right)$ decreases. There is also a critical value of $h$, labeled as $h_{c}$, where $I\left(h, \theta_{0}\right)$ vanishes. With these observations, we can conclude that the perturbation added at the left boundary will disrupt the wormhole geometry, and, as the wormhole geometry grows to a critical value, the mutual correlation vanishes for the left region and the right region is uncorrelated now.

For a fixed $h$, we also investigate the effect of the charge on the mutual correlation $I\left(h, \theta_{0}\right)$. Obviously, the larger the value of the charge is, the smaller the value of the mutual correlation $I\left(h, \theta_{0}\right)$ will be. This is similar to that of the static case in Section 3, for, in this case, the effect of the charge is dominated. The effect of the charge on the critical point $h_{c}$ is also investigated. The larger the value of the charge is, the smaller the value of the horizontal coordinate of the critical point $h_{c}$ will be. That is, in the shock wave geometry, the charge will prompt the correlated two quantum systems on the boundary of the AdS space-time to be uncorrelated.

\section{Conclusion and Discussion}

Usually, one often uses the mutual information, defined by the holographic entanglement entropy, to probe the entanglement of two regions living on the boundary of the AdS black holes. In [1], the author investigated the mutual information of the Reissner-Nordström AdS black holes with and without shock wave geometry. For the static case, they found that for large boundary regions the mutual information is positive while for small ones it vanishes. In the shock wave background, they found that the mutual information is disrupted by the perturbation added at the boundary, and, for large enough perturbation, the mutual information vanishes, which implies the left region and right region are uncorrelated.

In this paper, we employed the mutual correlation, which is defined by the geodesic length, to probe the correlation of two regions living on the boundary of the ReissnerNordström AdS black holes. We first investigated the mutual correlation in the static background. We found that as the size of the boundary region is large enough, the value of the mutual correlation is positive always, namely, the two regions living on the boundary of the AdS black holes are correlated. Our result implies that the mutual correlation has the same effect as that of the mutual information as they are used to probe the correlation of two regions. We also investigated the effect of the charge on the mutual correlation and found that it decreases as the charge increases. That is, the charge will destroy the correlation of correlated two regions.

By adding the perturbations into the bulk, we studied the dynamic mutual correlation in the shock wave geometry. We found that as the added perturbation becomes greater, the shift of the horizon becomes larger, and the mutual correlation decreases rapidly. In particular, there is a critical value for the shift where the mutual correlation vanishes as the perturbation is large enough. Obviously, our result is also the same as that probed by the mutual information in [1]. We also investigated the effect of the charge on the mutual correlation and found that the bigger the value of the charge is, the smaller the value of the mutual correlation will be. Namely, the charge will destroy the correlation of the correlated two regions, which is the same as that in the static background.

In [20], it has been found that, for a spin system, the two point functions and mutual information have a qualitatively similar response to a perturbation of the thermofield double state. Thus it is also interesting to use directly the two point functions to probe the butterfly effect though it is relatively cruder compared with the mutual information and mutual correlation [20].

\section{Data Availability}

All the figures can be obtained by the corresponding equations and values of the parameter. The authors did not adopt other data.

\section{Conflicts of Interest}

The authors declare that they have no conflicts of interest.

\section{Acknowledgments}

The authors are grateful to Hai-Qing Zhang for his instructive discussions. This work is supported by the National Natural Science Foundation of China (Grant no. 11405016) and Basic Research Project of Science and Technology Committee of Chongqing (Grant no. cstc2016jcyja0364). 


\section{References}

[1] S. Leichenauer, "Disrupting entanglement of black holes," Physical Review D: Particles, Fields, Gravitation and Cosmology, vol. 90, no. 4, 2014.

[2] D. Berenstein and A. M. Garcia-Garcia, "Universal quantum constraints on the butterfly effect," High Energy Physics, 2015.

[3] N. Sircar, J. Sonnenschein, and W. Tangarife, "Extending the scope of holographic mutual information and chaotic behavior," Journal of High Energy Physics, vol. 1605, no. 91, 2016.

[4] Y. Ling, P. Liu, and J.-P. Wu, "Holographic butterfly effect at quantum critical points," Journal of High Energy Physics, no. 10, 025, front matter+12 pages, 2017.

[5] Y. Ling, P. Liu, and J. Wu, "Note on the butterfly effect in holographic superconductor models," Physics Letters B, vol. 768, pp. 288-291, 2017.

[6] S. H. Shenker and D. Stanford, "Multiple shocks," Journal of High Energy Physics, vol. 2014, no. 12, 2014.

[7] D. A. Roberts, D. Stanford, and L. Susskind, "Localized shocks," Journal of High Energy Physics, vol. 1503, no. 51, 2015.

[8] S. H. Shenker and D. Stanford, "Stringy effects in scrambling," Journal of High Energy Physics, vol. 1505, 2015.

[9] X. Feng and H. Lü, "Butterfly velocity bound and reverse isoperimetric inequality," Physical Review D: Particles, Fields, Gravitation and Cosmology, vol. 95, no. 6, 2017.

[10] M. Alishahiha, A. Davody, A. Naseh, and S. F. Taghavi, "On butterfly effect in higher derivative gravities," Journal of High Energy Physics, vol. 32, 2016.

[11] A. P. Reynolds and S. F. Ross, "Butterflies with rotation and charge," Classical and Quantum Gravity, vol. 21, Article ID 215008, 2016.

[12] S. Grozdanov, K. Schalm, and V. Scopelliti, "Black Hole Scrambling from Hydrodynamics," Physical Review Letters, 2018.

[13] A. Lucas and J. Steinberg, "Charge diffusion and the butterfly effect in striped holographic matter," Journal of High Energy Physics, vol. 1610, no. 143, 2016.

[14] Y. Gu and X.-L. Qi, "Fractional statistics and the butterfly effect," Journal of High Energy Physics, vol. 1608, no. 129, 2016.

[15] R. Cai, X. Zeng, and H. Zhang, "Influence of inhomogeneities on holographic mutual information and butterfly effect," Journal of High Energy Physics, vol. 2017, no. 7, 2017.

[16] M. Maldacena, "Large N limit of superconformal field theories and supergravity," International Journal of Theoretical Physics, vol. 38, 1999.

[17] E. Witten, "Anti de Sitter space and holography," Advances in Theoretical and Mathematical Physics, vol. 2, no. 2, pp. 253-291, 1998.

[18] S. S. Gubser, I. R. Klebanov, and A. M. Polyakov, "Gauge theory correlators from non-critical string theory," Physics Letters B, vol. 428, no. 1-2, pp. 105-114, 1998.

[19] J. Maldacena and L. Susskind, "Cool horizons for entangled black holes," Fortschritte der Physik/Progress of Physics, vol. 61, 2013.

[20] S. H. Shenker and D. Stanford, "Black holes and the butterfly effect," Journal of High Energy Physics, vol. 1403, 2014.

[21] S. Ryu and T. Takayanagi, "Holographic derivation of entanglement entropy from AdS/CFT," Physics Letters B, 2006.

[22] J. Maldacena, S. H. Shenker, and D. Stanford, "A bound on chaos," Journal of High Energy Physics, vol. 1608, 2016.
[23] C. V. Johnson, "Large N Phase Transitions, Finite Volume, and Entanglement Entropy," Journal of High Energy Physics, vol. 1403, 2014.

[24] P. H. Nguyen, "An equal area law for the van der Waals transition of holographic entanglement entropy," Journal of High Energy Physics, vol. 12, p. 139, 2015.

[25] H.-L. Li, Z.-W. Feng, S.-Z. Yang, and X.-T. Zu, "Wilson loop's phase transition probed by non-local observable," Nuclear Physics, vol. 929, pp. 58-68, 2018.

[26] X. X. Zeng, H. B. Zhang, and L. F. Li, "Phase transition of holographic entanglement entropy in massive gravity," Physics Letters, vol. 756, pp. 170-179, 2016.

[27] X. X. Zeng and L.-F. Li, "Holographic phase transition probed by non-local observables," Advances in High Energy Physics, vol. 2016, pp. 1-13, 2016.

[28] S. He, L. F. Li, and X. X. Zeng, "Holographic van der waals-like phase transition in the Gauss-Bonnet gravity," Nuclear Physics B, vol. 915, pp. 243-261, 2017.

[29] A. Dey, S. Mahapatra, and T. Sarkar, "Thermodynamics and entanglement entropy with Weyl corrections," Physical Review D: Particles, Fields, Gravitation and Cosmology, vol. 94, 2016.

[30] X. X. Zeng and L. F. Li, "Van der waals phase transition in the framework of holography," Physics Letters B, vol. 764, pp. 100108,2017

[31] J. X. Mo, G. Q. Li, Z. T. Lin, and X. X. Zeng, "Van der Waals like behavior and equal area law of two point correlation function of $\mathrm{f}(\mathrm{R})$ AdS black holes," Nuclear Physics B, 2017.

[32] H. Liu and S. J. Suh, "Entanglement growth during thermalization in holographic systems," Physical Review D: Particles, Fields, Gravitation and Cosmology, vol. 89, no. 6, 2014.

[33] C. Park, "Holographic renormalization in dense medium," Advances in High Energy Physics, vol. 2014, 2014.

[34] X.-X. Zeng and W.-B. Liu, "Holographic thermalization in Gauss-Bonnet gravity," Physics Letters B, vol. 726, no. 1-3, pp. 481-487, 2013.

[35] X.-X. Zeng and W.-B. Liu, "Holographic thermalization with a chemical potential in Gauss-Bonnet gravity," Journal of High Energy Physics, vol. 3, no. 31, 2013.

[36] X. X. Zeng, D. Y. Chen, and L. F. Li, "Holographic thermalization and gravitational collapse in a spacetime dominated by quintessence dark energy," Physical Review D: Particles, Fields, Gravitation and Cosmology, vol. 91, 2015.

[37] X.-X. Zeng, X.-M. Liu, and W.-B. Liu, "Holographic thermalization in noncommutative geometry," Physics Letters B: Particle Physics, Nuclear Physics and Cosmology, vol. 744, pp. 48-54, 2015.

[38] X. X. Zeng, X. Y. Hu, and L. F. Li, "Effect of phantom dark energy on holographic thermalization," Chinese Physics Letters, vol. 34, 2017.

[39] Y. P. Hu, X. X. Zeng, and H. Q. Zhang, "Holographic Thermalization and Generalized Vaidya-AdS Solutions in Massive Gravity," Physics Letters B, vol. 765, pp. 120-126, 2017.

[40] H. Liu and S. J. Suh, "Entanglement tsunami: Universal scaling in holographic thermalization," Physical Review Letters, vol. 112, 2014.

[41] V. E. Hubeny, "Extremal surfaces as bulk probes in AdS/CFT," Journal of High Energy Physics, vol. 2012, no. 93, 2012. 

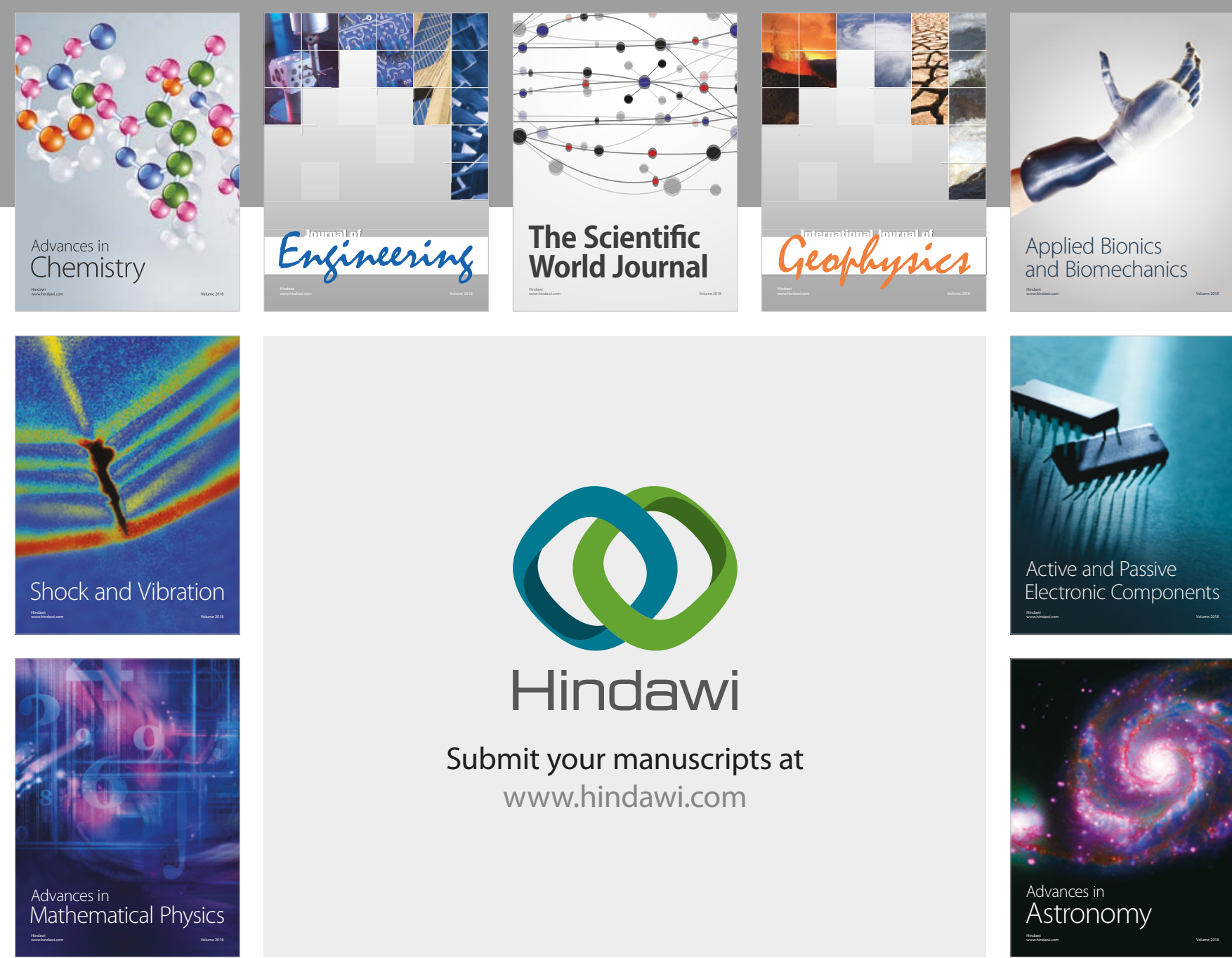

Submit your manuscripts at

www.hindawi.com

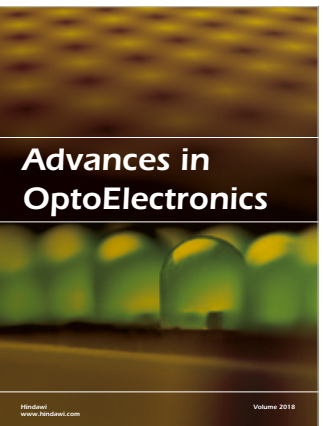

\section{Rotcting Machinery}
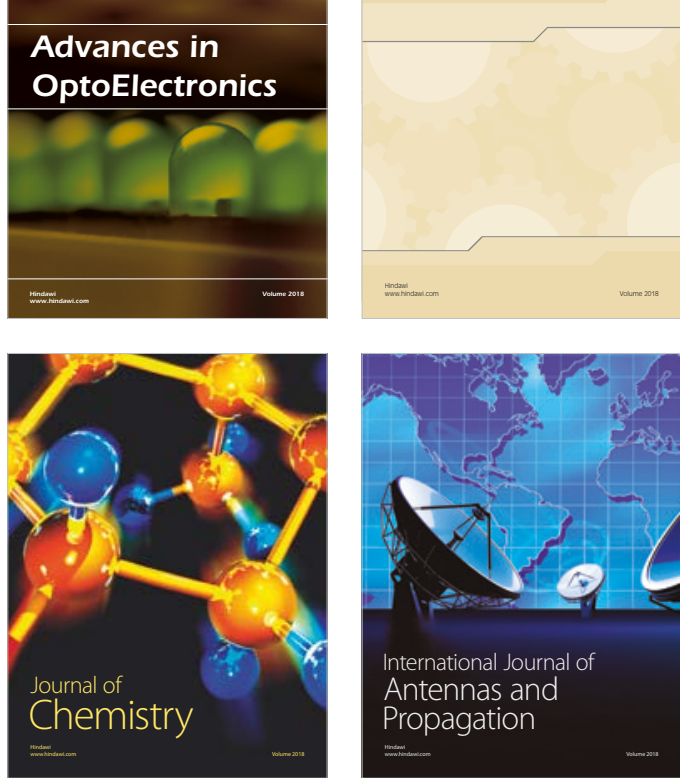

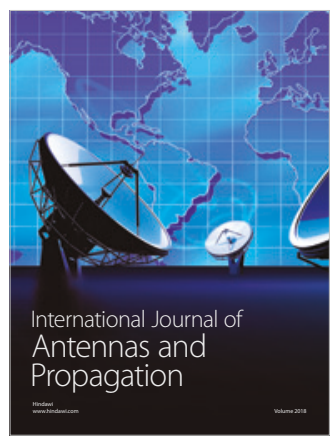

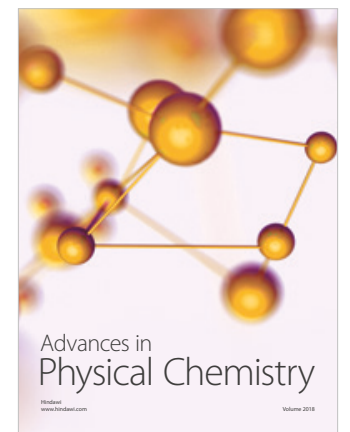

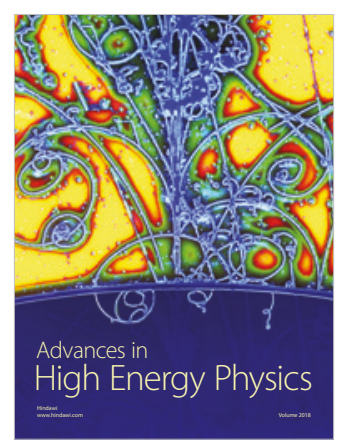

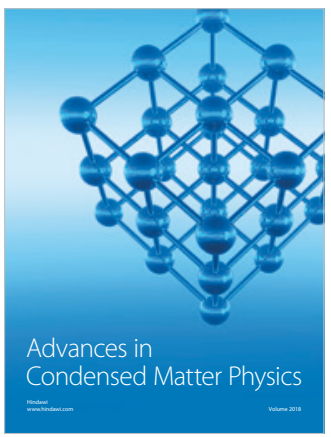

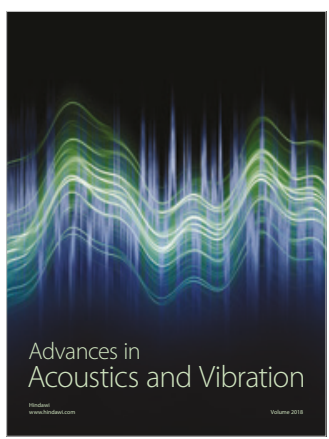

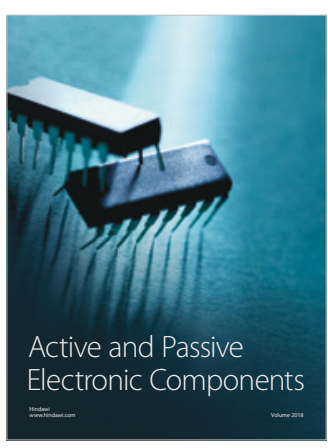
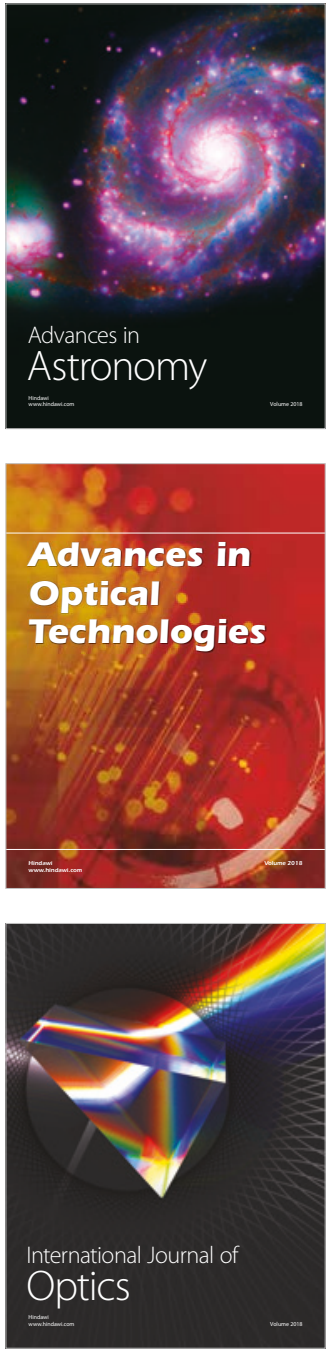